keitsarbeit von gemeinnützigen Organisationen. Verfügbar unter: http:// netzwerkpr.de/wp-content/uploads/2010/04/Kiefer_NGOs-im-SocialWeb.pdf [8.6.2012]

Kiefer, Kathrin (2012). NGOs im Social Web, Status Quo zum Social Media Einsatz in gemeinnützigen Organisationen. Verfügbar unter: http://netzwerkpr.de/index.php/2012/04/status-quo-zum-social-mediaeinsatz-in-npos/ [8.6.2012]

Kilian, T., Hass, B. \& Walsh, G. (2011). Grundlagen des Web 2.0. In Hass, B., Walsh, G. \& Kilian, T. (Hrsg.), Web 2.0 - Neue Perspektiven für Marketing und Medien, (3-20). Berlin: Springer.

King, H. (2012). Shitstorm. In Neues Wörterbuch der Szenesprachen. Verfügbar unter http://szenesprachenwiki.de/definition/shitstorm/ [8.6.2012]

Kühl, E. (2012). KONY 2012: Mit Social Media gegen den Völkermörder?. Verfügbar unter http://blog.zeit.de/netzfilmblog/2012/03/08/josephkony-2012-social-media-uganda-film/ [8.6.2012]

Lubasch, T., Böhm, F. \& Nöll, F. (2010). Social Media Report. Non-Profit-Organisationen starten in das Social Web. Spendino GmbH.

Reiser, B. (2012). Nonprofits müssen ins Internet und dieses mitgestalten. Blog noprofits-vernetzt.de. Verfügbar unter http://blog.nonprofitsvernetzt.de/index.php/nonprofits-mussen-ins-internet-und-dieses-mitgestalten/ [8.6.2012]

Schultz, F. \& Wehmeier, S.W. (2010). Online Relation. In: Schweiger, W. \& Beck K. (Hrsg.), Handbuch Online-Kommunikation (409-431). Wiesbaden: Verlag für Sozialwissenschaften.

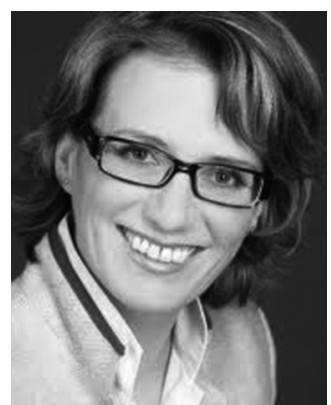

Kerstin Plehwe ist Vorsitzende der überparteilichen Initiative ProDialog Gesellschaft zur Förderung von Demokratie und Engagement $\mathrm{mbH}$ in Berlin. , gilt die Geschäftsführerin der Initiative ProDialog und Gründerin des Internationalen Instituts für Politik \& Gesellschaft als engagierte Protagonistin moderner Kommunikationswege und eines neuen Leadership-Verständnisses in Politik und Wirtschaft. E-Mail: plehwe@prodialog.org.

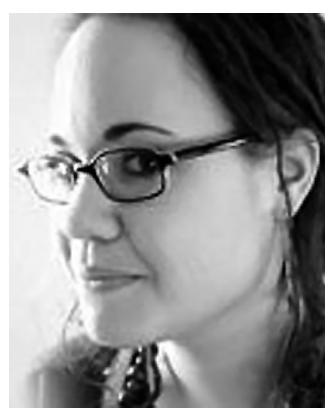

Sarah Bastgen ist Leiterin Politik und Gesellschaft der Initiative ProDialog und promoviert an der FU Berlin zum Thema Kommunikationsstrategien von NGOs im Europäischen Mehrebenensystem. Ihre Arbeitsschwerpunkte sind politische und zivilgesellschaftliche Kommunikation sowie europäische Zivilgesellschaft. E-Mail: bastgen@prodialog.org.

\title{
Die Transformation der öffentlichen Verwaltung durch die sozialen Medien
}

\author{
Boris von Chlebowski
}

\begin{abstract}
Kernaussagen
Mit Facebook, Twitter und Youtube haben die sozialen Medien die Art und Weise revolutioniert, wie Konsumenten und Bürger heutzutage Informationen sammeln, kommunizieren und zusammenarbeiten. Nachdem erfolgreiche Unternehmenskommunikation bereits seit längerem auf soziale Medien setzt, ist nun auch die öffentliche Verwaltung am Zug. Fest steht schon jetzt: Web 2.0 und die sozialen Medien werden die Arbeitsabläufe und Kultur der Verwaltung nachhaltig verändern.
\end{abstract}

\section{Revolutionen, Unternehmen und aktive Bürger}

\subsection{Die digitale Revolution}

Wenn man heute die Nachrichten verfolgt, ist nicht zu übersehen, welchen enormen Einfluss soziale Medien mittlerweile als Ausgangspunkt für Veränderungen haben. Die Menschen beziehen nicht nur ihre Nachrichten über soziale Medien, son- dern die sozialen Medien selbst stehen im Mittelpunkt der Nachrichten.

Nehmen wir zum Beispiel den Arabischen Frühling. In vielen Staaten Nordafrikas und des Nahen Ostens wurden die Regierungen ins Wanken gebracht - durch eine Massenbewegung, die größtenteils über soziale Medien initiiert und gelenkt wurde. Diese „E-Revolution“ hat sich im Laufe der Zeit über alle Grenzen hinweggesetzt, ob Klassen oder Geschlechter, politische oder nationale Territorien. Die Berichterstattung über die Ereignisse stützte sich in großen Teilen auf soziale Medien, die als Quelle für lokale Informationen dienten. So wurden aus persönlichen Schicksalen im Handumdrehen internationale Nachrichten.

Die öffentliche Verwaltung kann viel von den Ereignissen des Arabischen Frühlings, der Occupy-Bewegung oder aus „Stuttgart 21“ lernen. Jüngstes Beispiel ist das Anti-Produktpiraterie-Handelsabkommen ACTA, dessen spektakuläres Scheitern im Europäischen Parlament am 4. Juli 2012 wesentlich auf auf eine sorgfältig geplante Online- wie Offline-Kampagne zurückzuführen ist. Die Sozialen Medien und Web 2.0- 
Technologien machen die Menschen zu „digitalen Bürgern“. Durch die Nutzung komfortabler, einfach zugänglicher Technologien haben die Menschen heute jederzeit die Möglichkeit, sich zu äußern, Verbindung mit anderen Bürgern aufzunehmen und aktiv zu werden.

Das Radio benötigte 38 Jahre bis es eine Verbreitung bei 50 Millionen Nutzern fand. Facebook erreichte die gleiche Zahl an Nutzern in nur vier Jahren. Facebook hat eine Dimension erreicht, die gemessen an der Kopfzahl in einer Liga mit der Bevölkerung Indiens spielt. Dadurch entstehen zwangsläufig auch neue Wertschöpfungsmuster und neue Beteiligungsformen.

\subsection{Vorbild Unternehmenskommunikation}

Erfolgreiche Unternehmen haben längst erkannt, dass sich die Kommunikationssteuerung zu Gunsten der Community verlagert hat. Traditionelle und aufwendig inszenierte wie teure Marketingaktionen verlieren an Attraktivität, weil die Meinungsbildung der Konsumenten ohnehin anschließend in den Sozialen Netzwerken, also auf Facebook, Twitter und Youtube, abläuft. Erprobte Geschäftspraktiken müssen nach und nach überdacht werden, um die neuen Möglichkeiten von Social Media optimal für die Zwecke der Organisation zu nutzen. Die Ford Motor Company hat im Jahr 2011 erstmals ein neues Fahrzeug ausschließlich via Facebook vorgestellt. Dabei hat Ford mehr Kundenkontakte erzielt, als mit einem Fernsehspot vor dem Super Bowl. Ein Jahr zuvor hatte ein einziger solcher TV-Spot bei ähnlich Gelegenheit 3 Million Dollar für 30 Sekunden gekostet. Unternehmen, die gelernt haben die Chancen von Social Media für sich nutzen, profitieren erheblich. Aufwand und Ertrag von Investitionen in Social Media erreichen durchaus ein Verhältnis von 1:20.

\subsection{Von „Customer Insights“ zu „Citizen Insights”}

Unternehmen gehen dazu über, relevante Informationen interaktiv in Internetforen behutsam zu erfragen oder durch Mitlesen dazuzulernen. Laut einer gemeinsamen Untersuchung von SAS Institut und Accenture aus dem Jahr 2011 wollen 70 Prozent der US-Unternehmen in ihre analytischen Fähigkeiten investieren. 52 Prozent planen die Neueinstellung von DatenAnalysten. Die Möglichkeit Social Media auszuwerten, um z.B. eigene Marketingaktivitäten im Spiegel der Kundenkommentare auszuwerten, treiben diese Entwicklung. Im Vergleich zum traditionellen Sender-Empfänger-Schema erweist es sich auch als nachhaltiger und preisgünstiger, mitzubloggen, Wünsche aufzunehmen und gut dosiert auf Lob und Kritik zu reagieren. Transparenz und das Eingestehen von Fehlern heben dabei die Reputation beim Kunden.

Techniken, die im privatwirtschaftlichen Marketing entwickelt wurden, um ein besseres Marktverständnis zu erreichen und den Bedürfnissen potentieller Kunden gerecht zu werden, eignen sich prinzipiell auch für den Bürgerdialog. Sie könnten das Verhältnis staatlicher Entscheider zu ihren Bürgerinnen und Bürgern verbessern. Durch „Citizen Insights“ lässt sich im Verhältnis von Regierung und Bürger testen, wie groß z.B. die Akzeptanz für eine PKW-Maut tatsächlich ist. Wer die Diskurse auf Facebook auswertet, bekommt früher mit, ob die Bürger ihren Bahnhof lieber über oder unter der Erde haben wollen. Jedenfalls weitet sich der Blick dafür, welche Diskussionen jenseits der traditionellen Medien „im Schwange" sind.

\section{Was der digitale Bürger bewirkt}

Die digitalen Bürger werden auch einen großen Einfluss auf die tägliche Arbeit in der öffentlichen Verwaltung und auf die Erbringung öffentlicher Dienstleistungen ausüben. Auch wenn darüber nicht in den politischen Blogs, den Abendnachrichten oder den Geschichtsbüchern berichtet wird - die digitalen Bürger sorgen dafür, dass sich die alltäglichen Abläufe in der öffentlichen Verwaltung unwiderruflich verändern. Accenture hat im Jahre 2012 im Rahmen einer Studie Bürger in sieben Ländern befragt und herausgefunden, dass die Mehrheit der Befragten das Angebot digitaler Dienstleistungen in der öffentlichen Verwaltung nutzen würde, insbesondere bei Standardvorgängen. Darüber hinaus sind 51 Prozent der Befragten davon überzeugt, dass die Möglichkeit, über digitale Kanäle mit der öffentlichen Verwaltung zu kommunizieren, ihr Verhältnis zum Staat und seinen Institutionen verbessern würde.

Bei diesen Ergebnissen fühlen sich vielleicht einige Beobachter der IT-Entwicklung im öffentlichen Bereich an die Anfänge von E-Government erinnert. Die Diskussion drehte sich damals um die Frage, ob E-Government (von statischen Websites bis zu interaktiven Portalen) das Potenzial hätte, das Verhältnis zwischen Regierung und Wählern zu verändern. Heute kann diese Frage eindeutig mit „Ja“ beantwortet werden.

Aber anders als damals bestimmen die digitalen Bürger heute in einem nie gekannten Ausmaß das Kräftespiel zwischen Staat und Bürgern - was durchaus positiv zu sehen ist. Die digitalen Bürger von heute sind in der Lage, ihren Erwartungen an den Staat Ausdruck zu verleihen und sich so schlagkräftig zu organisieren, dass der Staat nicht anders kann, als darauf zu reagieren. Der Staat muss sich heute die zentrale Frage stellen, welche Veränderungen auf seiner Seite notwendig sind, um die Beziehung zu den Bürgern zu verbessern. Einige wesentliche Punkte sind offenkundig:

1. Nutzer-Erwartungen wandeln sich radikal: $\mathrm{Zu}$ beobachten ist ein Trend, wonach sich die Bürgerinnen und Bürger zunehmend auch außerhalb von Wahlen in politische und Verwaltungsentscheidungen einbringen möchten. Der Siegeszug von Social Media nährt die Erwartung, dass auch die Verwaltung online in Echtzeit informiert, dialogisch kommuniziert und Abstimmungen durchführt. Verwaltungsdaten sollen zugänglicher und transparenter gemacht werden. Die Ansprüche an die Informationspolitik und die Dialogbereitschaft der Behörden wächst. Dabei ist Social Media das Medium der Wahl, wenn ein lebendigerer Dialog ermöglicht werden soll. Die Akzeptanz ist hier deshalb besonders hoch, weil Facebook 
\& Co. eine nichthierarchische Form des Bürgerdialogs darstellt. Unattraktiv sind dagegen alle archaischen Formen nach dem Motto „Ich Sender - Du Empfänger!“

2. Zeit ist ein zentraler Faktor: In der digitalen Welt ist Zeit ein entscheidender Aspekt. Die digitalen Bürger können blitzschnell etwas bewirken, und sie erwarten eine entsprechende Reaktionsgeschwindigkeit zunehmend auch vom Staat. 2011 haben in Spanien Tausende junger Leute gegen Massenarbeitslosigkeit, Sparmaßnahmen und politische Korruption protestiert. Die Bewegung begann als Flashmob auf der Puerta del Sol in Madrid am 15. Mai 2011 und breitete sich sehr schnell aus. Diese Ereignisse hatten einen signifikanten Einfluss auf die Wahlbeteilung bei der Parlamentswahl im November. Die regierenden Sozialisten mussten ihre schlimmste Wahlniederlage in mehr als 30 Jahren hinnehmen.

3. Der Staat muss den Menschen folgen: Die digitalen Bürger sind überall in sozialen Netzwerken unterwegs. Der Staat muss dort ebenfalls präsent sein, um mit ihnen in Kontakt zu bleiben. Diese Erkenntnis wurde in den USA bei den Präsidentschaftswahlen 2008 umgesetzt. Viele Beobachter sind der Ansicht, dass der damalige Kandidat Barack Obama deshalb so erfolgreich Spenden sammeln und Wähler mobilisieren konnte, weil sein Team das Internet auf intelligente Weise genutzt hat - vor allem auch, um mit jüngeren Wählern in Kontakt zu kommen. Nachdem er gewählt war, hat Barack Obama als erster amerikanischer Präsident eine Twitter Town Hall initiiert. Soziale Medien sind sehr populär, und der Staat muss Fähigkeiten in diesem Bereich aufbauen, um mit den Menschen interagieren und sie als eine Quelle für Feedback und Innovationen nutzen zu können. Entscheidend wird jedoch sein, dass der Staat den richtigen Gebrauch von diesem Interaktionskanal macht und die Anliegen der Bürger versteht. 51 Prozent der Befragten in der oben genannten Studie erklärten, dass sie nicht abgeneigt wären, über soziale Medien Kontakt zu einem Beamten oder Angestellten aufzunehmen, um etwas zu beantragen oder eine Lösung für ein Problem zu finden.

4. Öffnung bedeutet mehr Beteiligung: Unternehmerische Initiativen entwickeln sich zu einem wichtigen Thema für die öffentliche Verwaltung. Einige Staaten haben bereits damit angefangen, ihre Daten, Prozesse und Leistungserbringung öffentlich zugänglich zu machen, um die Bürger stärker einzubinden und neue Entwicklungen zu ermöglichen. Mit diesen Schritten wird dem engen Zusammenhang zwischen sozialen Medien und Transparenz in der öffentlichen Verwaltung Rechnung getragen. Großbritannien und Dänemark haben beispielsweise eine Flut öffentlicher Daten gebündelt verfügbar gemacht, um die Bürger in Entscheidungsprozesse zu untegrieren. Die Herausforderung besteht hier in der effektiven Handhabung des öffentlichen Engagements und im bestmöglichen Einsatz von neuen Technologien im Rahmen der betreffenden IT-Initiativen.

5. Veränderungen fangen im Kleinen an: Soziale Medien werden die persönliche Interaktion nicht vollständig ersetzen - jedenfalls nicht in absehbarer Zeit. Es wird sich auch erst nach und nach zeigen, ob dieser Interaktionskanal langfristig genutzt wird und wie sich seine Nutzung im Laufe der Zeit verändert. Feststeht, dass der Staat bei der Erbringung von öffentlichen Dienstleistungen die richtigen Kanäle für eine wirksame Interaktion mit seinen Bürgern auswählen muss. Dabei spielen auch Faktoren wie Zugangsmöglichkeiten, kulturelle Normen und zur Verfügung stehende Mittel eine wichtige Rolle. Neben der Weiterentwicklung digitaler Kanäle werden auch persönliche Kontakte und Telefongespräche weiterhin von Bedeutung sein. Auf die Frage nach ihrem bevorzugten zukünftigen Kommunikationsweg mit der öffentlichen Verwaltung antworteten 60 Prozent der Teilnehmer, dass sie auch in Zukunft immer noch am liebsten mit einem Beamten oder Angestellten persönlich oder am Telefon sprechen würden, um ein Problem zu lösen. Dennoch muss der Staat verstehen: Die Menschen werden durch Tablet-PCs, Internetverbindungen und neue Technologien zu digitalen Bürgern - und die ganze Welt ist für sie zum Greifen nah.

\section{Der Fortschritt ist eine Schnecke}

\subsection{Ein Anfang ist gemacht}

Der Trend hat inzwischen auch den deutschen Public Sector erreicht. Die vom FAZ-Institut herausgebrachte Forsa-Studie „Branchenkompass 2012 Public Services“ bestätigt erneut, dass öffentliche Verwaltungen eine stärkere Bürgerbeteiligung durch soziale Medien anstreben und es liegt nahe, dass sich mit Social Media Angeboten die Bürgerbeteiligung und Servicequalität in deutschen Behörden verbessern lässt.

In der Bundesverwaltung sind es gerade die jüngeren Minister und Staatssekretäre, die das Thema forcieren. Wenn sie bereits privat oder als Abgeordnete auf Facebook sind, so erreichen sie über diesen Kanal zwangsläufig auch Nachrichten und Kommentare, die sie in ihrer Regierungsfunktion betreffen. Einfach „Wegklicken“ ist dann keine Option.

Auch die Kommunen entdecken das Instrument und nutzen es strategisch: Die Freie und Hansestadt Hamburg hat mit dem Stadtportal hamburg.de eine attraktive Plattform, die viel zum Image einer modernen Medienmetropole beiträgt. Hamburg ist die Stadt mit den meisten Followern bei Facebook: fast 700.000 Personen „gefällt das“ (Juli 2012).

In der niederrheinischen Stadt Moers ziehen städtische Internetredaktion, Pressestelle und kommunales Rechenzentrum an einem Strang, um ihre bundesweite Spitzenstellung bei der Social-Media-Nutzung zu verteidigen. Für eine Stadt mit gut rund 100.000 Einwohnern sind mehr als 3.000 Fans bei Facebook, 700 Follower bei Twitter und 100 Abonnenten bei YouTube beeindruckende Werte.

\subsection{Viele Fragen offen}

Trotz dieser Beispiele und einer zunehmenden Erwartungshaltung haben bislang nur wenige Behörden eigene Erfahrungen mit elektronischer Beteiligung von Bürgern an politischen Entscheidungsverfahren oder mit Behördenkommunikation via 
Social Media. Die meisten Behörden sind bei der Wahl ihrer Instrumente des Bürgerdialogs eher konservativ. Auf den Internetseiten sieht man oft nur ganz einfache Kontaktformulare. Gelegentlich findet man etwas offenere Foren. Eine Minderheit folgt dem Beispiel der Bundeskanzlerin und richtet sich mit Video-Podcast an die Öffentlichkeit. Die ganze Bandbreite der Möglichkeiten wird selten genutzt. Das gilt insbesondere für die sozialen Netzwerke wie Facebook oder Xing. Noch weniger sind bei Twitter unterwegs.

Die Dinge sind noch sehr im Fluss. Es herrscht große Unsicherheit darüber, wie man das Web 2.0 richtig nutzt. Dabei ist es sicher so, dass die Kommunikationsagenturen ihren Regierungskunden sehr gerne mehr innovative Kommunikationswege eröffnen würden. Gleichzeitig ist die Nutzung von Social Media durch Unternehmen nicht 1:1 auf die Verwaltung übertragbar. Stattdessen müssen gewisse Besonderheiten und Eigengesetzlichkeiten des öffentlichen Sektors berücksichtigt werden.

Die Behörden haben aber schon in der ersten Generation des Internets gelernt: Kommunikationsplattformen darf man nicht sich selbst überlassen. Sie müssen gepflegt werden. Es genügt nicht, ein Online-Portal lediglich zur Verfügung zu stellen. Foren bedürfen der Moderation. Digitale Medien verlangen ein ehrgeizigeres Antwortzeitverhalten, als das in der traditionellen Behördenkommunikation üblich war. Zumindest in den Ministerien sind eigene online-Redaktionen entstanden. Deren Personalbedarf unterscheidet sich aber erheblich, je nachdem ob dort nur Pressemitteilungen ins Netz gestellt werden oder wirklich interaktive Zwei-Wege-Kommunikation aktiv angeregt und zielgruppenspezifisch gesteuert werden soll. Social Media lässt sich nicht mal eben nebenher von der Pressestelle miterledigen.

\section{Die Social-Media-Agenda der öffentlichen Verwaltung}

Weil solche Vorfragen unklar sind, drücken sich viele Behörden noch um eine explizite Social-Media-Strategie herum. Tatsächlich führt aber gar kein Weg daran vorbei sich zu überlegen, welche Ziele man im Web 2.0 erreichen will und wo dabei das Anwendungsfeld für Social Media genau liegen soll.

Viel hängt davon ab, ob die neuen Kommunikationsprozesse sorgfältig geplant werden. Ordnung kommt erst in die Sache, wenn strategisch entschieden ist, welche interaktiven Online-Dienste eine Behörde für welche Inhalte und Zielgruppen nutzen möchte. Dabei gilt es dann, sich auf die Eigenart dieser Medien auch tatsächlich einzulassen: Keine wirksame Unterstützung der Öffentlichkeitsarbeit ist es sicherlich, wenn Twitter einfach nur zum Versenden der üblichen Pressemitteilungen verwendet wird, ohne dem personalisierten und interaktiven Charakter des Mediums gerecht zu werden.

\section{1 „Ohne Facebook“ ist auch keine Lösung}

Wie so oft bei technischen Neuerungen, begegnet man skeptischen Traditionalisten. Sie argumentieren häufig damit, dass sie zahlreichen Widrigkeiten aus dem Wege gehen, wenn sie auf das neue Instrument verzichten. Diese Denkweise hat manches für sich. Zugleich vernachlässigt sie aber die Tatsache, dass auch der Verzicht auf eine Social-Media-Strategie seine Konsequenzen hat. In einem solchen Dilemma steckt derzeit beispielsweise die Polizei. In Hannover gefällt die örtliche Polizei 100.000 Facebook-Mitgliedern. Alleine im August 2011 verzeichnete die Behörde mehr als 2,2 Millionen Zugriffe auf ihre Facebook-Seite. Bestimmte Meldungen wurden bis zu 150.000 Mal gelesen. Das bringt fassbare Erfolge: In wenigen Monaten konnten auf diese Weise allein in Hannover acht Fahndungen erfolgreich abgeschlossen werden. Darunter zwei vermisste Kinder. Über die klassischen Medien war im selben Zeitraum kein einziger entscheidender Tipp gekommen. Auf solche Instrumente verzichtet man ungern. Auch wenn der niedersächsische Landesdatenschutzbeauftragte es mit Besorgnis sieht, dass die Datenspuren deutscher Ermittlungsarbeit auf Servern außerhalb der EU liegen.

\subsection{Es zählen Relevanz und Glaubwürdigkeit}

Wir sehen in der Welt der Unternehmen, dass Social Media ihre Kommunikation informeller und authentischer gemacht hat. Es wird sich zeigen, ob in Politik und Verwaltung ein ähnlicher Wandel einsetzen wird. Für die weitere Entwicklung kommt es darauf an, ob nur die technischen Möglichkeiten von Social Media zunehmende Verbreitung im Bürgerdialog finden, oder ob sich die dahinter stehenden Beteiligungsoptionen auch qualitativ verändern. Das berührt die Gretchenfrage der Relevanz solcher Angebote. Die Nutzer haben einen feinen Sinn dafür, ob Online-Dialoge nur Partizipation vorgaukeln oder ob die Argumente tatsächlich in Entscheidungsprozesse einfließen. Der Lackmustest ist oft bereits die innerbehördliche Aufhängung des Themas. Wird Social Media als bloßer Appendix der Presse- und Öffentlichkeitsarbeit behandelt oder gibt es auch eine institutionelle Anbindung an die fachliche Expertise?

\subsection{Den kulturelle Unterschied verstehen}

Die Berliner Kommunikationsberaterin Ute Pannen (2011) hat darauf hingewiesen, dass die Unterschiede zwischen der „Kultur des Internets“ und der „Kultur eines Ministeriums“ kaum größer sein könnten. Sie schildert, wie schwer externen Kommunikationsagenturen die Sisyphusarbeit fällt, diese Kulturen zu synchronisieren: In der hierarchisch organisierten Verwaltung geht es um Rechtmäßigkeit und Legitimation durch ein zentralisiertes Verfahren, Mitzeichnung aller mitbetroffenen Arbeitseinheiten. Gründlichkeit geht vor Schnelligkeit. Social Media ist dagegen dezentral und an Communities orientiert. Transparenz und Aktualität sind tendenziell wichtiger als Sachrationalität im Detail.

Ein traditioneller Antwortentwurf für einen Minister durchläuft mindestens vier Hierarchiestufen. Der zugehörige Deckvermerk ist beim Eingang im Ministerbüro mit Paraphen, Anmerkungen und Ergänzungsvorschlägen oft geradezu übersät. Dagegen kommt bei schneller online-Kommunikation 
dem einzelnen Bearbeiter in der Web-Redaktion der Behörde eine Schlüsselrolle zu. Ihn muss man durch ein geeignetes Wissensmanagement unterstützen. Diese Mitarbeiter müssen kommunikationsstarke Generalisten sein, die klug genug sind, im richtigen Moment auch einmal besonders vorsichtig zu formulieren. Naive Aktivitäten im Web 2.0 werden dagegen rasch zum Fiasko.

Dabei spielt eine Rolle, dass in Deutschland nicht nur förmliche Verwaltungsakte, sondern auch sog. Realakte wie behördliche Auskünfte, oder Warnungen justiziabel sind. Wenn ein einfacher Abgeordneter sich zweifelnd über die Qualität eines bestimmten Lebensmittels äußert, so bleibt das in der Regel ohne juristische Folgen. Bezeichnet aber ein Regierungspräsident oder ein Landesverbraucherschutzminister ein Lebensmittel als „unappetitlich“, so kann ein Staathaftungsprozess in Millionenhöhe durchaus die Folge sein. Das muss man wissen, wenn man für einen Verbraucherschutzminister twittert.

\subsection{Das Personal ist der Erfolgsfaktor}

Eine gewisse Offenheit für die neuen Technologie dürfte Staat und Verwaltung auch im „War for Talents“ zu Gute kommen: Innerhalb der nächsten zehn Jahre werden über eine Million Beschäftigte der öffentlichen Verwaltung von Bund und Ländern die Altersgrenze erreichen und aus dem Dienst ausscheiden. Der Verwaltungsnachwuchs geht ganz selbstverständlich mit Twitter, Skype und Facebook um. „E-Mail-Ausdrucker“ gelten ihnen als die „Schatten-Parker“ des 21. Jahrhunderts. Manche Neueinstellung im öffentlichen Dienst ereilt heute eine Art Kulturschock, wenn sich im neuen Job die papiergebundenen Vorgänge auf Aktenböcken stapeln. Wir wissen aus der Fachkräftegewinnung in der Wirtschaft, dass eine zeitgemäße, technisch moderne Arbeitsumgebung ein wichtiger Faktor ist, wenn es um die Attraktivität eines Arbeitsplatzes geht.

$\mathrm{Da}$ ist es kein Wunder, dass schon die ersten öffentlichen Stellen beginnen, massiv über Facebook zu rekrutieren. Nehmen wir die Bundeswehr: Mindestens 15.000 junge Leute will die Bundeswehr jedes Jahr als Berufs- und Zeitsoldaten gewinnen und wünscht sich für eine Stelle mehrere Bewerber. Ohne den Einsatz von Social Media kommt man schwer an die Zielgruppe der Schulabgänger und Arbeitsuchenden im Alter zwischen 17 und 30 Jahren heran. Deshalb hat das Verteidigungsministerium hier merklich investiert. Inzwischen schneidet die Facebook-Karriereseite der Bundeswehr im Ranking besser ab als die Karriereseiten von Top-Unternehmen wie Audi, Lufthansa oder Bayer.

Und ein weiterer Aspekt: bevor sich eine Behörde auf die Nutzung von Social Media einlässt, sollte sie unbedingt die betreffenden Mitarbeiterinnen und Mitarbeiter eingehend schulen, wie man mit Bürgern und Unternehmen online kommuniziert und professionell mit Kritik umgeht. Bisher gibt es noch $\mathrm{zu}$ wenig wirklich gute Weiterbildungsangebote für Behördenmitarbeiter auf diesem Gebiet.

Vorbildlich ist hier etwa die Finanzbehörde der Freien und Hansestadt Hamburg: Mit ihrem Leitfaden „Social Media in der Hamburgischen Verwaltung “ will sie ihren Verwaltungsmitarbeitern erste Orientierungen vermitteln, sich in der Fülle der Möglichkeiten von Social Media zurechtzufinden, den für den individuellen Bedarf einer Behörde richtigen Ansatz auszuwählen und ihn unter den speziellen Gegebenheiten der öffentlichen Verwaltung erfolgreich in die organisatorischen Prozesse zu integrieren.

\section{Soziale Medien strategisch einbinden}

Die öffentliche Verwaltung wird vom Engagement in den sozialen Medien profitieren, wenn sie diese - über die externe Kommunikation hinausgehend - als ein strategisches Instrument versteht, um ihre Leistungen und die Art ihrer Leistungserbringung zu verbessern. Darüber hinaus sollte sie die technischen Möglichkeiten als Chance zur Steigerung der eigenen Attraktivität bei der Personalgewinnung einsetzen und für den internen Informationsaustausch nutzen - und das am besten behördenübergreifend.

Souveränität im Umgang mit den sozialen Netzwerken werden die Behörden entwickeln, wenn sie sich die verschiedenen Plattformen genau anschauen und entscheiden, welche für sie relevant sind. Auf dieser Grundlage sollten sie eine Social-Media-Strategie entwickeln und diese in die Gesamtstrategie und Abläufe ihrer Organisation integrieren. Erfolgsentscheidend wird jedoch, daß die öffentliche Verwaltung die Wirkungen ihrer Social-Media-Aktivitäten misst und steuert, denn ohne den Nachweis sinnvoller Wirkungen werden Investitionen in Social Media nur schwer zu begründen sein.

Ferner muss die Verwaltung dafür sorgen, dass sich ihre Social-Media-Strategie in die übergeordnete IT- bzw. EGovernment-Strategie einfügt und anknüpfende Fragestellungen beantwortet wie beispielsweise die Auswertung großer strukturierter und unstrukturierter Datenmengen (Big Data). Und schließlich ist auch hier zu fordern, daß die öffentliche Verwaltung ihre Social-Media-Strategie und deren Umsetzung möglichst ebenenübergreifend angeht, Strukturen und Abläufe standardisiert und das Prinzip der Shared Services anwendet.

\section{Literatur}

Pannen, Ute. (2011). Das Internet ist kein Zauberstab. Berliner Republik 4/11.

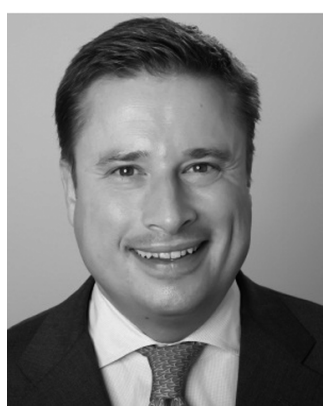

Boris von Chlebowski, Diplom-Kaufmann, ist als Mitglied der Geschäftsführung bei Accenture, verantwortlich für die Kontakte zu Entscheidungsträgern in Politik und Verwaltung auf Bundes- und Landesebene. Email: boris.chlebowski@accenture.com 